\title{
Sulforaphane-cyclodextrin Complex
}

National Cancer Institute

\section{Source}

National Cancer Institute. Sulforaphane-cyclodextrin Complex. NCI Thesaurus. Code

C113804.

An orally available, stable powder formulation composed of sulforaphane, a naturallyoccurring phytochemical belong ing to the class of isothiocyanates, encapsulated within alpha-cyclodextrin, with chemopreventive activity. Upon administration of sulforaphane/alpha-cyclodextrin complex SFX-01, sulforaphane activates the transcription factor NF-E2-related factor 2 (Nrf2; NFE2L2), a member of the basic leucine zipper family, which binds to and activates antioxidant-response elements (AREs). Subsequently, activated AREs mediate the transcription of various cytoprotective genes and antioxidant enzymes, particularly phase II detoxification enzymes, such as glutathione-S-transferase and quinone oxidoreductase, resulting in the detoxification of highly reactive carcinogens. Cyclodextrin is able to prevent sulforaphane's rapid breakdown, which improves its stability and half-life. 